\title{
Cryptococcal Meningitis in a HIV-Negative Patient
}

\author{
Cátia Barreiros, Lúcia Meireles-Brandão, Duarte Silva, Carmélia Rodrigues, Diana Guerra \\ Internal Medicine Department, Serviço de Medicina 1, Unidade Local de Saúde do Alto Minho, EPE, Portugal
}

Received: 09/11/2017

Accepted: 20/11/2017

Published: $12 / 12 / 2017$

How to cite this article: Barreiros C, Meireles-Brandão L, Silva D, Rodrigues C, Guerra D. Cryptococcal meningitis in a HIV-negative patient. EJCRIM 2017;4: doi:10.12890/2017_000778.

Conflicts of Interests: The Authors declare that there are no competing interests.

This article is licensed under a Commons Attribution Non-Commercial 4.0 License

\section{ABSTRACT}

Sarcoidosis is a risk factor for the development of cryptococcal infection due to dysfunction at T-cell level. Its rarity may, however, delay diagnosis and treatment. We describe the case of a 60-year-old man, diagnosed with sarcoidosis since 1999. He had never received systemic immunomodulatory therapy, such as corticosteroid therapy. In 2012, he was diagnosed with pulmonary cryptococcosis and treated with fluconazole. In April 2013, he presented with symptoms compatible with central nervous system (CNS) infection, namely, Cryptococcus neoformans meningitis. He was treated with amphotericin B, followed by fluconazole. The clinical outcome was favourable.

\section{LEARNING POINTS}

- Although rare, in patients with sarcoidosis and central nervous system (CNS) symptomatology, it is important to verify the existence of cryptococcal meningitis.

- Antifungal treatment should be started as early as possible.

- Before the diagnosis of pulmonary cryptococcosis, dissemination to the CNS should be outruled due to the need for more aggressive treatment.

\section{KEYWORDS}

Sarcoidosis, Cryptococcus neoformans, meningitis, HIV negative

\section{INTRODUCTION}

Cryptococcal meningitis, an opportunistic infection often found in HIV-positive patients, also occurs in immunosuppressive conditions such as long-term treatment with corticosteroids, transplanted patients and oncological patients, predominantly haematological. In the case of sarcoidosis, it is a rare complication that must be considered, and which is explained by T-cell dysfunction. T-cell immunity is the main route of protection against cryptococcal infections ${ }^{[1,2]}$.

\section{CASE DESCRIPTION}

In our case, we describe a 60-year-old man diagnosed with stage IV sarcoidosis since 1999, with no history of systemic corticosteroid therapy or HIV. A lung biopsy performed in May 2012 showed 'fibrous and inflammatory changes of interstitial lung disease and very suspect findings of sarcoidosis-non-necrotizing granulomas'. He typically presented with medium-effort dyspnea and was treated with inhaled salmeterol and fluticasone.

In June 2012, he was diagnosed with pulmonary cryptococcosis, confirmed by the identification of Cryptococcus neoformans in bronchial 


\section{of Case Reports in \\ Internal Medicine}

secretions. He was treated with fluconazole for 6 months and involvement of the central nervous system (CNS) was not excluded at the time. In April 2013, he was observed with periorbital and left frontotemporal headache, fever $\left(38^{\circ}-39^{\circ} \mathrm{C}\right)$, asthenia, anorexia and dry cough, having already been treated with amoxicillin/clavulanic acid but without improvement. There was no evidence of nausea, vomiting, visual changes or hearing loss. He was orientated and cooperative, showing neck stiffness, incoordination in the heel-knee test, and an ataxic gait. Fever $\left(38.7^{\circ} \mathrm{C}\right)$ and scattered wheezes throughout the right hemithorax were present. Analytically, no leucopenia or lymphopenia were found (Table 1). A cranial CT scan showed right frontal hypodensity, with nonspecific characteristics, which may explain an old ischaemic lesion. A lumbar puncture showed lymphocytic pleocytosis, hypoglycorrhachia and hyperproteinorraquia (Table 2). China ink staining was negative for Cryptococcus neoformans and capsular antigens were also negative. A chest X-ray showed bilateral and parahilar heterogeneous hypotransparencies, similar to previous X-rays (Fig. 1).

Having considered infection of the CNS, we initiated empirical therapy with ampicillin and ceftriaxone. On day 2, the patient still had a fever so the lumbar puncture was repeated, which revealed 50 leucocytes (Table 2). The search for Cryptococcus neoformans by China ink staining was negative once again. However, taking into account the previous diagnosis of pulmonary cryptococcosis, we decided to initiate treatment with conventional amphotericin B. Meanwhile, the cerebrospinal fluid (CSF) culture was positive for Cryptococcus neoformans, confirming cryptococcal meningitis. Hepatitis B, C and HIV serology with HIV PCR were negative (Table 1). The patient completed 4 weeks of amphotericin $\mathrm{B}$ and had a positive clinical course. Ambulatory treatment with fluconazole was maintained.

\begin{tabular}{|l|l|l|}
\hline Tests & Reference values & $13 / 04 / 2013$ \\
\hline Haemoglobin (g/dL) & $11.8-15.8$ & 12.7 \\
\hline Leucocytes (uL) & $4.0-10$ & 6320 \\
\hline Neutrophils & $1800-7700$ & $4370(69.2 \%)$ \\
\hline Lymphocytes & $800-4000$ & $1130(17.9 \%)$ \\
\hline Platelets (10//uL) & $150-400$ & 232 \\
\hline Creatinine (mg/dL) & $0.6-1.0$ & 0.91 \\
\hline $\begin{array}{l}\text { Sodium/Potassium } \\
\text { (mmol/L) }\end{array}$ & $136-145 / 3.5-5.1$ & $127 / 4.3$ \\
\hline Total bilirubin (mg/dL) & $0.3-1.2$ & 0.87 \\
\hline $\begin{array}{l}\text { Aspartate } \\
\text { aminotransferase/ } \\
\text { Alanine transaminase (UI/L) }\end{array}$ & $8-35 / 7-45$ & $17 / 13$ \\
\hline $\begin{array}{l}\text { Alkaline phosphatase/ } \\
\text { Gamma glutamyl } \\
\text { transferase (UI/L) }\end{array}$ & $30-120 /<38$ & $67 / 28$ \\
\hline $\begin{array}{l}\text { Lactate dehydrogenase } \\
\text { (Ul/L) }\end{array}$ & $125-220$ & 215 \\
\hline Sedimentation rate (mm/h) & $4-10$ & 103 \\
\hline $\begin{array}{l}\text { Serum lgG/lgA/lgM (mg/dL) } \\
700-1600 / \\
60-400 / 40-230\end{array}$ & Normal \\
\hline $\begin{array}{l}90-180 / \\
12-40 / 23-46\end{array}$ & Normal \\
\hline Blood cultures & Negative \\
\hline VHB, VHC, HIV1e2* & Negative \\
\hline PCR test HIV1e2 & Negative \\
\hline
\end{tabular}

Table 1. Analytical study

*Hepatitis B, C and HIV serology

\begin{tabular}{|l|l|l|}
\hline Tests & $13 / 04 / 2013$ & $15 / 04 / 2013$ \\
\hline Total cells (mm3) & 28 & 150 \\
\hline Leucocytes (mm3) & 28 & 50 \\
\hline Neutrophils (\%) & 46 & 78 \\
\hline Lymphocytes (\%) & 54 & 22 \\
\hline Glucose (mg/dL) & 44 & 48 \\
\hline Proteins (mg/dL) & 66 & 62.5 \\
\hline $\begin{array}{l}\text { Adenosine deaminase } \\
\text { (U/L) }\end{array}$ & 6.4 & 6.0 \\
\hline
\end{tabular}

Table 2. Serial CSF analysis

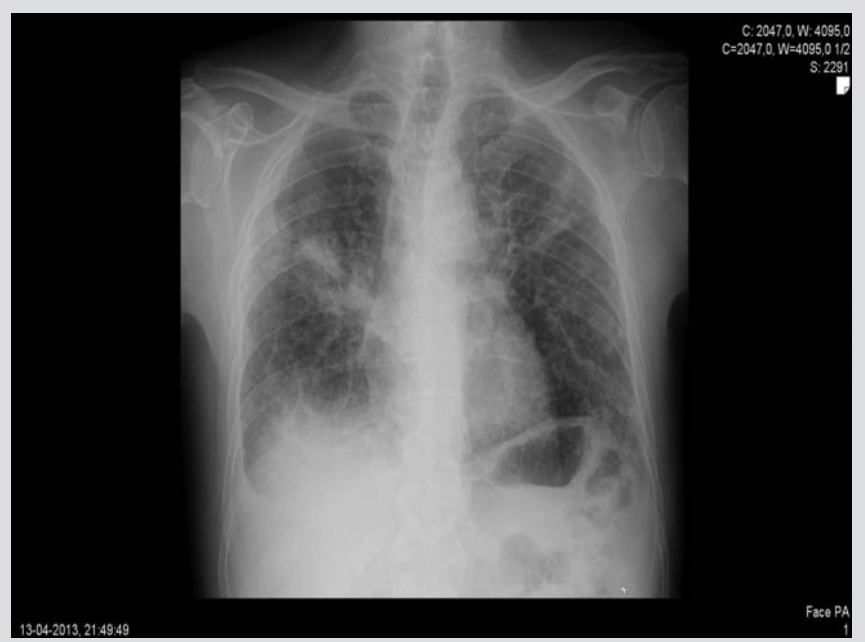

Figure 1. Chest $x$-ray 


\section{DISCUSSION}

Although rare, sarcoidosis is known to be an independent risk factor for cryptococcal disease, even when not associated with corticosteroid therapy or extrapulmonary disease, as in the case of this patient $t^{[3]}$. With a recent diagnosis of pulmonary cryptococcosis and the onset of headache and neurological changes, it was essential to consider the cryptococcal meningitis hypothesis. It should be noted that the microbiological diagnosis is not always linear, sometimes being necessary to repeat exams.

In the present case, the therapy was initiated before obtaining the result of the cultural examination, which was probably a decisive factor concerning its prognosis. As evidenced in several cases, the early initiation of treatment has prognostic implications ${ }^{[1]}$. It should be noted that when the diagnosis of pulmonary cryptococcosis was made, dissemination to the CNS should had been excluded due to the need for more aggressive treatment.

In a review of the literature conducted in 2016 by Leonhard et al., they found that the mean age of patients with sarcoidosis who developed cryptococcal meningitis was 39 years old and that $40 \%$ of patients were undergoing immunomodulatory therapy ${ }^{[1]}$. Our patient was well above this age group and has never been treated with systemic corticosteroid or other immunomodulators, which makes this case of particular interest.

\section{REFERENCES}

1. Leonhard S, Fritz D, van de Beek D, Brouwer M. Cryptococcal meningitis complicating sarcoidosis. Medicine 2016;95:e4587.

2. Bernard C, Maucort-Boulch D, Varron L, Charlier C, Sitbon K, Freymond N, et al. Cryptococcosis in sarcoidosis: cryptOsarc, a comparative study of 18 cases. Q J Med 2013;106:523-39.

3. Adams T, Gibson M. Cryptococcal meningitis in a patient with sarcoidosis. Proc (Bayl Univ Med Cent) 2016;29:207-08. 